FSS

Romero Saletti, S. M., Van den Broucke, S., \& Chau, C. (2021). The effectiveness of prevention programs for problematic Internet use in adolescents and youths: A systematic review and meta-analysis. Cyberpsychology: Journal of Psychosocial Research on Cyberspace, 15(2), Article 10. https://doi.org/10.5817/CP2021-2-10

\title{
The Effectiveness of Prevention Programs for Problematic Internet Use in Adolescents and Youths: A Systematic Review and Meta-Analysis
}

\author{
Silvana M. Romero Saletti ${ }^{1}$, Stephan Van den Broucke' ${ }^{1}$ \& Cecilia Chau² \\ ${ }^{1}$ Université catholique de Louvain, Louvain-la-Neuve, Belgium \\ ${ }^{2}$ Pontificia Universidad Católica del Perú, Lima, Peru
}

\begin{abstract}
Problematic Internet use (PIU) can lead to dysfunction and undesired consequences, especially in adolescents and youths. Preventive interventions can reduce them, but should be built on sound evidence. This review synthesizes the available evidence on the effectiveness of primary prevention programs for PIU in adolescents and youths. It adds to previous reviews by broadening the search to general and specific problematic behavior. Two independent reviewers performed a systematic search for published studies on PIU and internet addiction in English, French and Spanish using PubMed, PsycINFO, Cochrane Register of Controlled Trials, and Scopus. Published experimental and quasi-experimental studies that assessed the effectiveness of primary prevention programs targeting PIU behaviors were considered for inclusion. Full texts for eligible studies were retrieved and assessed for quality. Five studies were retained for narrative synthesis. Three of them based the intervention strategy on an underlying theory, one on media literacy, and one used an educational-based approach. A meta-analysis showed that all five interventions were effective in preventing internet addiction separately. However, when using the Hartung-KnappSidik-Jonkman (HKSJ) estimator and removing one of the studies because of a disproportionate Hedges' $g$, combined effects were no longer significant. Although PIU is a popular topic, little is known about ways to prevent it. This review demonstrates that prevention programs can be based on different theoretical approaches, but that the available evidence is too heterogeneous to derive generalizable conclusions concerning their effectiveness.
\end{abstract}

Keywords: Problematic internet use; internet addiction; primary prevention; adolescents; youths

\section{Introduction}

The increased availability of Internet and its extended use through smartphones has given rise to concerns about their effect on wellbeing. Adolescents (12-17 years) and emerging adults (18-29 years) seem particularly vulnerable since they access the internet more than other age groups (Anderson et al., 2017). Roughly two-thirds of the world's population who use the internet are among the 15-24 age group (Kardefelt-Winther et al., 2019), and in Europe, 95\% of individuals between 16-29 access the internet daily (EUROSTAT, 2020). As "digital natives", youths rely on the internet for performing various day-to-day tasks, for information, knowledge exchange and study, and for socializing, getting entertained, and playing (D'Hondt et al., 2015; Kardefelt-Winther et al., 2019; Wallace, 2014). Consequently, youths become more reliant on visual communication, are eager for instant gratification, and are more likely to engage in risky behaviors due to technology misuse (Teo, 2013).

Using the Internet brings many benefits to the majority of its users. A recent literature review report concerning children up to 18 years old suggested that moderate use of digital technology tends to be beneficial for their mental wellbeing and stimulating for their social relationships (Kardefelt-Winther, 2017b). Nonetheless, its 
excessive, uncontrolled, or compulsive use can lead to psychological ailments such as depression, anxiety, stress, and loneliness (Elhai et al., 2017; Kim et al., 2009). Other undesired consequences have also been reported, such as impaired academic and working performance, sleep deprivation, family problems, and reduced social activities (Wallace, 2014). In turn, these problems result in high public health and societal costs (World Health Organization, 2015).

\section{What Constitutes Problematic Internet Use?}

Maladaptive use of the Internet can be conceptualized as a behavioral addiction. It has been referred to by various terms, including internet addiction (IA), compulsive internet use, internet dependence, and pathological internet use (Spada, 2014; Volpe et al., 2015). This conceptualization, however, remains controversial (D'Hondt et al., 2015; Starcevic, 2013), since it is based on confirmative approaches (Billieux et al., 2015) which over-pathologize the condition by applying substance use criteria without distinguishing a broader spectrum of behaviors. For instance, no clear distinction is made between high involvement versus dysfunctional involvement. As long as the debate continues on what constitutes a behavioral addiction (Kardefelt-Winther, 2014), whether researchers may be "overpathologizing" everyday life behaviors, or whether the internet is not just a vehicle that fuels disordered behaviors (Griffiths, 2018), the use of the addiction framework and criteria borrowed from substance or gambling addictions fail to explain why internet-related conditions occur and persist (Kardefelt-Winther, 2017a).

As an alternative, the term Problematic internet use (PIU) has been suggested. This umbrella term (Fineberg et al., 2018) describes a maladaptive pattern of internet use that involves an apparent loss of control over the behavior, the occurrence of psychological, social, or professional negative consequences and worries, and obsessive thoughts when it is not possible to use the internet (D'Hondt et al., 2015). The term includes, but is not limited to, online gaming, gambling, buying, and pornography viewing, as well as social networking and cyberbullying (Fineberg et al., 2018).

Some clinical aspects of these behaviors resemble those of addictions. This is particularly the case for gambling and pornography viewing, which involve impaired control over the behavior, craving, persistence despite damaging effects, and preoccupation with the associated functional impairment. However, it is unclear whether all PIU behaviors meet addiction's physiological criteria, such as increased tolerance and withdrawal (Volpe et al., 2015). Some PIU behaviors have been claimed to share similarities with symptoms of other disorders such as obsessive-compulsive disorders, anxiety disorders, and impulse control disorders (Spada, 2014; Volpe et al., 2015).

\section{Problematic Internet Use as a Public Health Concern}

Given the difficulties of defining and operationalizing PIU's phenomenon, it is not surprising that prevalence rates vary significantly (Mihajlov \& Vejmelka, 2017). Reported rates among adolescents and college students from China, South Korea, the United States, and European countries vary from 1 to 26\% (Rumpf et al., 2014; Wu et al., 2013). Regarding gender differences in IA tendencies, a recent meta-analysis comprising 115 independent samples involving 204,352 participants from 34 countries found a small effect of gender, implying that men tend to have more IA symptoms than women. This effect was greater for Asian countries. Meta-regression revealed smaller effect sizes in countries with higher GDP per capita and higher internet penetration. The authors point out that although they used general IA measures in their meta-analysis, gender may have different effect sizes both in magnitude and directionality for specific behaviors such as gaming (where men could show higher IA symptoms) and social networking (where women could show higher symptoms) (Su et al., 2019).

In line with the conceptualization of PIU as a behavioral addiction or other disorders, clinical interventions for PIU and IA have been developed and implemented, most of which are based on therapeutic and pharmacological strategies that are commonly used for substance disorders, obsessive-compulsive disorder and impulse control disorders (Mihajlov \& Vejmelka, 2017). A meta-analysis of 16 studies with 670 participants found that psychological and pharmacological interventions effectively improve IA symptoms, reduce the time spent online, and decrease depression and anxiety (Winkler et al., 2013).

While the effectiveness of treating PIU and IA thus seems increasingly well documented, the evidence regarding the ways to prevent PIU and to promote adequate Internet use is much less well developed. Nevertheless, the topic 
is high on the public health agenda. An international meeting on the public health implications of excessive use of the internet, computers, smartphones, and similar electronic devices organized by the World Health Organization in 2014 reached the conclusion that there is a need to: (1) define the scope, phenomenology, and typology of PIU; (2) intensify international research to address current knowledge gaps, generate essential information for the development of PIU prevention and treatment policies; and (3) document and evaluate policies, strategies, and interventions to prevent and reduce health risks and disorders associated with PIU (World Health Organization, 2015). In line with these recommendations, it is essential to address the gaps between what we know about PIU and what researchers and clinicians actually do to prevent PIU.

\section{Prevention Programs for PIU}

To our knowledge, three published studies exist that have used the PRISMA guidelines for systematic reviews to document the prevention of PIU. The first one (Throuvala et al., 2019) sought to identify prevention programs or protocols for IA targeting adolescents in the school context, examine their effectiveness and highlight their strengths, limitations and best practices. Fifteen intervention studies were included in the review, but due to the heterogeneity of their scope, content, outcome evaluation, and assessment tools used, the findings regarding the effectiveness of the programs remained inconclusive. The second review (Bağatarhan \& Siyez, 2015) focused on the effectiveness of prevention programs for internet addiction during adolescence, drawing on multiple research databases in Turkish and English. Of the 435 articles found, only 5 met the inclusion criteria. However, as the only search terms used for this review's outcomes were "problematic internet use", and "internet addiction", it is likely that some relevant articles were overlooked. The third review (Vondráčková \& Gabrhelík, 2016), conducted in Spanish and English, used more specific search terms to target the different PIU behaviors, such as: "gaming addiction", "sex addiction", and "social network addiction". This review could trace 179 articles published between January 1995 and April 2016, but only six of them described and evaluated specific prevention interventions. All three reviews agree that the literature on the prevention of internet addiction and PIU is scarce and of limited quality. Particular points of criticism are the absence of consensual diagnostic criteria, which leads to a diversity of IA/gaming assessment tools used; the various methodological limitations in the programs' designs; and the use of the time spent on internet/games as an outcome variable (Throuvala et al., 2019). The reviews also agree that there is a need to introduce and implement interventions for at-risk populations and effectiveness evaluations using well-designed studies. None of the reviews used meta-analysis to synthesize the findings.

The more consistent use of robust outcome measures is encouraged when selecting a measure that will prove the effectiveness of a preventive intervention since it can enable comparison of results across studies and allow for easier consolidation of findings (Breedvelt et al., 2020; Fineberg et al., 2018). Nonetheless, as mentioned before, there is quite some diversity regarding PIU conceptualization, assessment and various outcome measures could be used in primary prevention studies. First, questionnaires designed to measure PIU or IA symptoms, such as the well-established Young's Internet Addiction Test (Young, 1998) or similar. This could also include questionnaires designed for specific behaviors such as gaming and gambling (for a list, see Fineberg et al., 2018). Although not reliable as a standalone measure, many researchers attempt to quantify screen time, time spent online, duration, or frequency of internet access as PIU measures (Throuvala et al., 2019). Finally, other common outcomes that can be measured in primary prevention programs outside behavior are: knowledge, attitudes, and skills, and their measurement will depend on the complexity of the program and whether it is theory-driven or not (Leschied et al., 2018). Despite this could pose a problem when trying to synthesize the results from empiric research, in order to address this variety, various outcome measures could be included in our selection criteria.

The present systematic review aims to address these points by investigating the state of published research investigating the effectiveness of primary prevention programs for PIU. To that effect, multiple databases were searched using both general terms (e.g., "compulsive internet use", "internet addiction") and specific terms (e.g., "gaming addiction", "online gambling") targeting the different behaviors that are comprised by PIU in both adolescents and youths. Since a lack of consensus about terms is one of the most pressing concerns in this field, using a wide variety of terms referring to PIU behaviors allows finding more relevant studies and enables to map which behaviors are most often targeted in preventive interventions. In addition, it allows investigating how authors conceptualize PIU, which risk and protective factors are addressed, which strategies are used, and what evidence exists regarding their effectiveness. 


\section{Methods}

This systematic review was conducted following the PRISMA guidelines for systematic reviews. This review's protocol was registered in the PROSPERO database of prospectively registered systematic reviews (CRD42019135887).

\section{Eligibility Criteria}

To be included in this review, studies needed to meet the following criteria:

1. Be published studies concerning primary prevention programs for PIU or Internet Addiction in general or address specific behaviors that PIU comprises, such as problematic smartphone use, problematic social networking, gaming disorder, cybersex addiction, online gambling, and online buying.

2. Their target population should be adolescents and youths aged 12 to 29 years old, as these are the groups that report most PIU behaviors and access the internet more than other age groups (Anderson et al., 2017)

3. The studies need to provide a quantitative evaluation of the program's effectiveness by measuring at least one of these possible outcomes: a) symptom reduction outcomes using a valid and reliable questionnaire such as Young's Internet Addiction Test or questionnaires designed to measure specific behaviors (gaming, gambling, etc.); b) usage outcomes that quantify time spent online, duration or frequency; c) knowledge, attitude or awareness levels shifts; d) skill enhancement improvement outcomes such as self-efficacy, self-control, problem-solving, critical thinking, etc.

4. Be published in English, Spanish, and French, since those are the authors' native or expert languages.

Conversely, studies were excluded when they:

1. Were not concerned with primary prevention programs and the assessment of their effectiveness as their primary objective.

2. Addressed cyberbullying, which is an extension of bullying usually studied on its own and with the particularity of being a behavior perpetrated with the intention to harm, of repetitive nature and with a clear power imbalance between the aggressor and the victim (Tokunaga, 2010). The effectiveness of cyberbullying preventive interventions has been extensively and systematically reviewed over the past few years (Gaffney et al., 2019; Tanrikulu, 2018).

3. Were concerned with early intervention, treatment or rehabilitation instead of primary prevention.

4. Did not have a control group in the evaluation design.

\section{Search Strategy and Information Sources}

A three-step search strategy was used to locate published research. First, an initial limited search of Pubmed and PsychInfo was undertaken to identify keywords and index terms. Next, a second search was performed by two researchers across PubMed, PsycINFO, Cochrane Register of Controlled Trials and Scopus, using the following keywords for the targeted behaviors: "problematic internet us*", "compulsive internet us*", "pathological internet us*", "excessive computer us*", "internet addiction", "smartphone addiction", "cellphone addiction", "excessive mobile phone us*", "problematic smartphone us*", "problematic mobile phone us*", "problematic online gaming", "internet gaming", "Online gaming", "gaming addiction", "internet gaming addiction", "internet gaming disorder", "gaming disorder", "problematic social media us*", "problematic social networking us*", "social network addiction", "facebook addic*", "instagram addiction", "problematic social networking", "internet sex addiction", "cybersex", "online sex addiction", "online pornography", "compulsive online pornography viewing", "online gambling", "problematic online gambling", "problematic internet gambling", "problematic online shopping", "compulsive online shopping". These were combined with the targeted intervention key words: "prevention", "prevent", "prevention program", "psychoeducation", "online program" and with the targeted population: "adolesc*", "youth*", "teen*", "university student*", "undergraduate student*", "college student*". The search was limited to articles in English, French and Spanish and no date limits were imposed. The search strategy was adapted for each database to account for differences in terminology and indexing and was carried out between August and 
September of 2019. Finally, the reference list of all studies selected for critical appraisal was examined to seek for additional studies.

\section{Study Selection and Data Collection Process}

Figure 1. PRISMA Flow Chart of the Selection Process.

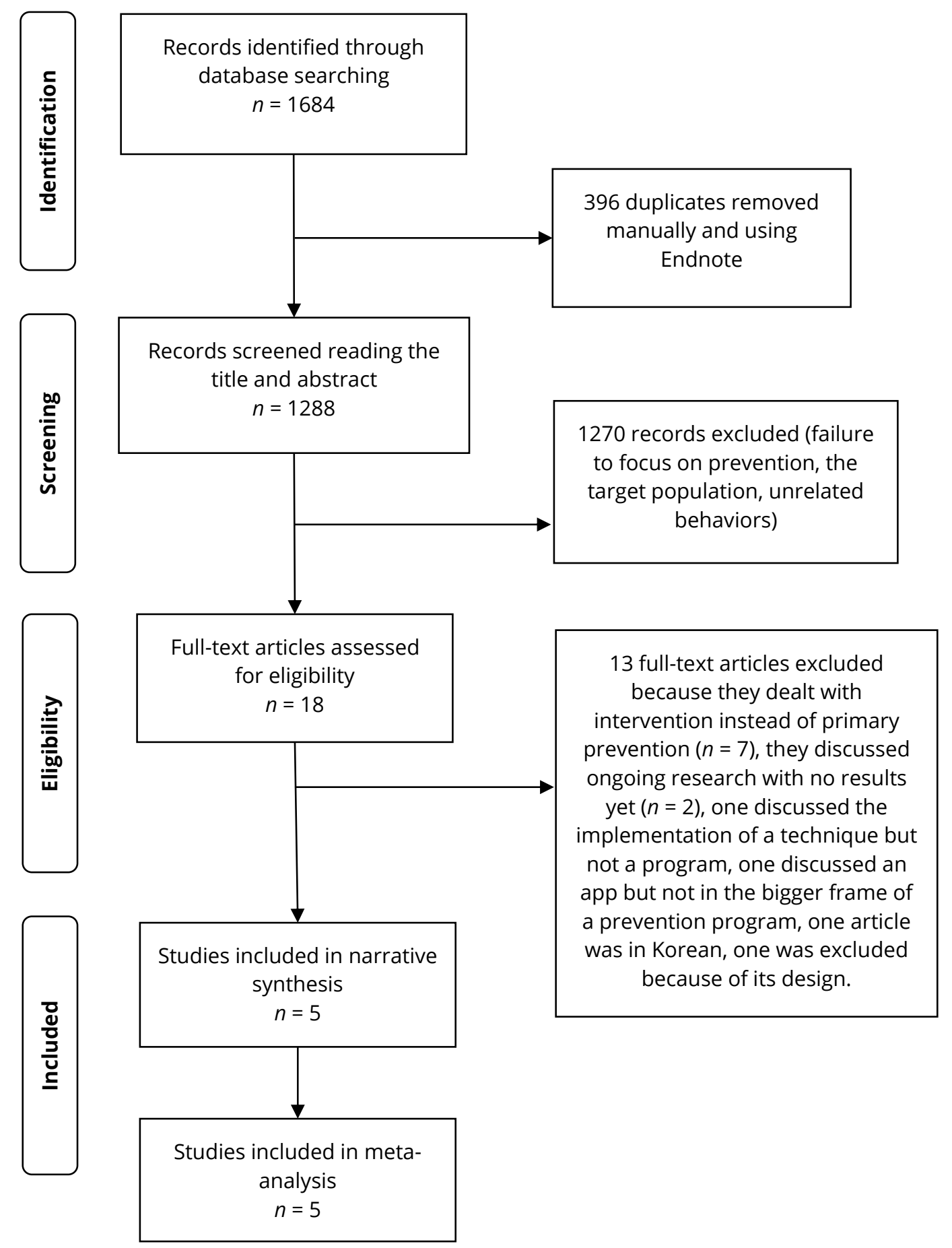

The search of all the databases yielded 1684 records (see Figure 1). After removing duplicates with Endnote and manually, 1288 records remained. These were screened by one researcher based on the titles and abstracts. The vast majority were related to the targeted behaviors but did not discuss prevention programs or their effectiveness and, as such, failed to meet the inclusion criteria. Following their removal, 18 articles were retained, retrieved in full, and assessed for eligibility by reading the full text by a second researcher, yielding a high agreement on inclusion (Cohen's kappa $=0.91$ ). Doubts regarding the possibility of including an article were resolved through discussion and agreement with a third researcher. Of the 18 articles, 13 were excluded for various reasons (see Table 1): 7 were concerned with therapeutic interventions rather than primary prevention, 2 discussed ongoing research with no results yet, 1 discussed the implementation of a technique (journaling), 1 discussed an app to 
imagine consequences of excessive smartphone use, 1 was only available in Korean, and 1 compared two interventions but did not use a control group. As a result, 5 studies were retained for quality assessment.

Table 1. Summary of Excluded Studies and Main Reasons for Exclusion.

\section{Author (year) \\ Country \\ Program name}

1. Benson et al. (2014)

USA

[no name]

2. Chow et al. (2018)

Hong Kong

Time Off

3. Fontalba-Navas et al. (2015)

Spain

[no name]

4. Gaysina \& Zakirova (2017)

Russia

[no name]

5. Joo \& Park (2010)

Korea

Empowerment Education

Program

6. Ke \& Wong (2018b)

Malaysia

Internet use for youth

7. Ke \& Wong (2018a)

Malaysia

Internet Use for Youth

8. Lee et al. (2016)

Korea

[no name]

9. Lindenberg et al. (2017)

Germany

PROTECT

10. Marco \& Chóliz (2017)

Spain

Design

Prevtec 3.1

11. Odacı \& Çelik (2017)

Turkey

[no name]

12. Shek \& Leung (2013)

Hong Kong

Youngster Internet Addiction

Prevention and Counseling

Service

13. Soto et al. (2018)

Spain

[no name]
Main reason for

exclusion

\section{Explanation}

Treatment, not prevention

The study tests the efficacy of a group treatment model for compulsive buying disorder using cognitive-behavioral, dialectical-behavioral and psychodynamic psychotherapy plus psychoeducation, motivational interviewing, acceptance and commitment therapy, and mindfulness.

Treatment, not prevention The study conveys a field trial of a character-based mobile application to stimulate imagination and reflect on the consequences of excessive use of smartphones in adolescents.

No results available

Treatment, not prevention

The study aims to develop an intervention model for PIU for high school adolescents. The authors claim to have carried out the program on 26 occasions in 22 schools and 1200 students, but no results are available. The study aims to present a model of psycho-pedagogical prevention of Internet addiction for 10 to 15-year-old students but assesses their internet addiction instead. Therefore it is not focused on primary prevention. Doubts arise regarding quality.

The study aims to determine the effects of an empowerment education

Article in Korean program on internet games addiction, empowerment, and stress in middle school students, using a non-equivalent, control group pretest-posttest design.

The study aims to develop a program based on cognitive-behavioral Treatment, not prevention therapy by addressing negative coping styles to reduce PIU in adolescents. One hundred fifty-seven participants aged 13 to 18 completed eight weekly, 90 min sessions, showing improvement after the program.

This study designs and tests a preventive intervention program based on Treatment, not prevention cognitive behavioral therapy for youths with PIU. A total of 45 secondary students from four schools completed the program, which was conducted in a group format by registered school counselors.

Not a program, relies on one strategy: journaling

Treatment, not prevention The study aims to assess the therapeutic effectiveness of a home-based daily journal of smartphone use in Korean adolescents to reduce smartphone addiction.

The study seeks to develop and test an indicated preventive intervention to reduce IUD (Internet Use Disorder) using a cognitive-behavioral, 4-session brief protocol with 340 students aged 12-18.

The study seeks to prevent techno-addictions, particularly gaming, by establishing limits and boundaries on the time spent online and gaming, organizing free time, and communicating appropriately with parents. Nonetheless, the study designed compared the traditional version of the program with an enhanced version that introduces impulse control techniques. One thousand one hundred ten students aged 9-16 were recruited.

Treatment, not prevention The study seeks to test the efficacy of a reality therapy-based group counseling on college students' PIU and life satisfaction. 20 students out of 418 met the criteria for PIU and were treated.

The study portrays the main features of a counseling model for Internet

Treatment, not prevention Addiction in adolescents drawing from motivational interviewing, family perspectives, and multi-level counseling at the individual, peer, and family levels.

The study presents the experience of a treatment center in Malaga at developing a proposal for an integrated approach for treating dysfunctional uses of ICT (Information and communication technologies). This approach includes both preventive strategies aimed at schools and treatment both at the individual and family level.

\section{Quality Assessment}

Downs and Black's (1998) checklist was used to assess the quality of the studies considered for inclusion in this review. This tool has 27 items designed to provide an overall score of quality as well as sub-scores for: reporting quality, external validity, internal validity (study bias, confounding and selection bias) and power. The advantage of this tool over others is that it can be used to assess both randomized and nonrandomized health care 
interventions. To ensure that a sufficient number of studies could be retained for the analysis, a criterion of $60 \%$ positive answers was established, meaning that only medium to high-quality studies were included in the review.

\section{Data Extraction and Analysis}

For the narrative synthesis, relevant data were extracted for the following categories: authors, year, country, program name, participant's characteristics, intervention characteristics (aim, theoretical background and key strategies used), measures used, and main findings. For the meta-analysis, quantitative data were pooled in RStudio using the meta package, version 4.9-7. Since prevention programs often occur in school settings, the randomization to conditions was sometimes carried out at the classroom or school level, enhancing the chance of underestimating the within-study variance. Therefore, it is necessary to adjust the variance of effect sizes to account for clustering (Donner \& Klar, 2002; Higgins \& Green, 2008). This can be achieved by estimating a design effect using the formula:

Design effect $=1+(M-1) \times I C C$

where $M$ is the mean cluster size in each study (mean number of students per classroom) and ICC the intraclass correlation coefficient, which was established at 0.025 following recommendations based on previous metaanalysis of school-based interventions (Gaffney et al., 2019). The variance can be multiplied by the design effect to obtain the corrected variance. This correction was applied to the raw data were necessary before pooling it for the meta-analysis. Separate effect sizes regarding Internet addiction were calculated for the post measures for the intervention and control groups, since they were measured using similar scales in all the studies. Not all studies reported follow-up measures, so these could not be included as outcome variables for the analysis. Screen time and self-reported internet use measured by hours or days a week are not reliable, and were therefore not used either.

As an estimator of the effect size, Hedges' $g$ 95\% confidence interval was used. Hedge's $g$ is preferred over Cohen's $d$ since it corrects for bias due to small sample sizes (Hedges \& Olkin, 1984). Its interpretation is similar to Cohen's criteria, distinguishing between small (.20), medium (.50), and large (.80) effect sizes. A random-effects model was used for the analysis. This model assumes that the effects estimated in the studies are not identical but follow some distribution (Higgins \& Green, 2008). This is the most appropriate approach as it cannot be assumed that the studies included were functionally identical (Borenstein et al., 2009). To estimate the variance of the distribution of true effect sizes, denoted by $\tau^{2}$, the Hartung-Knapp-Sidik-Jonkmann (HKSJ, Hartung \& Knapp, 2001) method was used along with the more well-known DerSimonian-Laird estimator (Dersimonian \& Laird, 1986), which is prone to producing false positives, especially when the number of studies included in the meta-analysis is small and where there is high heterogeneity. The HKSJ method has shown to outperform the DerSimonian-Laird method in many cases and is known for producing more conservative results shown in wider confidence intervals (IntHout et al., 2014). Finally, to assess heterogeneity, which is the extent to which effect sizes vary within a metaanalysis, we used $R$ (Higgins \& Thompson, 2002), derived from Cochran's $Q$ statistic and is the percentage of variability in effect sizes not caused by sampling error. Values of $R^{2}$ are interpreted as follows: $R=25 \%$, low heterogeneity; $l^{2}=50 \%$, moderate heterogeneity; $l^{2}=75 \%$, high heterogeneity. If high heterogeneity is observed, an influence analysis based on graphs can be conducted to detect outliers that could influence the pooled effect estimate (Viechtbauer \& Cheung, 2010) and the Leave-one-out analysis. The number of studies was too small to perform an Egger's test and further methods to assess publication bias.

\section{Results}

\section{Quality Assessment}

The five studies that met the inclusion criteria obtained the minimum of $60 \%$ required to assure quality. Overall, the studies obtained good scores for general quality ( $M=21$ out of 27 with $77 \%$ positive answers). The score for reporting quality was high ( $M=8.6$ out of 10 , with $86 \%$ of positive answers), as well as for internal validity bias ( $M$ $=4.8$ out of 7 , with $69 \%$ of positive answers). Average scores were obtained for external validity ( $M=2.4$ out of 3 , with $80 \%$ of positive answers) and internal validity confounding ( $M=4.2$ out of 6 , with $70 \%$ positive answers). Four of the five studies gave information about the sample size calculation or power estimations. 


\section{Narrative Synthesis}

The five studies that met the selection criteria aimed at preventing Internet Addiction (IA) in general. No published primary prevention programs were found to specifically address the problematic use of social networks, cybersex addiction, online gambling, online gaming, or online buying that met the criteria. A summary of participant characteristics, intervention characteristics, and main findings is given in Table 2.

Table 2. Summary of Studies Included for the Narrative Synthesis of Prevention Programs for PIU.

\section{Author \\ (year) \\ Country \\ Intervention}

\section{Maheri et $\mathbf{1 6 0}$ female}

al. (2017)

Iran

[no name] medical college students

$(M=21.8$ years old, randomly assigned to CG/IG)

$\begin{array}{ll}\text { 2. Uysal \& } & \mathbf{8 4} \text { students } \\ \text { Balci (2018) } & \text { aged 11-16 } \\ \text { Turkey } & \text { (randomly } \\ \text { Healthy } & \text { assigned to } \\ \text { Internet Use } & \text { CG/IG) } \\ \text { Program } & \end{array}$

3. Walther et al. (2014) Germany Connected www.worlds

1879 students aged 10 to 14 (cluster-level randomized to CG/IG)

$\begin{array}{ll}\text { 4. S.-Y. Yang } & \mathbf{7 9} \text { students } \\ \text { \& Kim } & \begin{array}{l}\text { aged 13-15 } \\ \text { (randomly } \\ \text { (2018) }\end{array} \\ \text { South Korea } & \text { assigned to } \\ \text { [no name] } & \text { CG/IG) }\end{array}$

5. X.-H. Yang et al.

(2019)

China [no name]
532 undergrad students 16-24. (randomly assigned to CG/IG)

Intervention Characteristics

Main findings

Aim: to increase knowledge of internet addiction, conscience of negative consequences of internet use, acknowledge the benefits of adopting preventive behaviors and overcoming barriers to preventive behaviors

Theory: Health Belief Model.

Duration: 3 sessions/3 weeks

Techniques: group discussions and lectures.

Outcome measures: HBM constructs (knowledge,

susceptibility, perceived severity, perceived barriers, perceived benefits, cause to action, self-efficacy), Internet addiction (IAT), Hours of internet use per day

Aim: to reduce PIU by targeting personal (healthy internet use habits), behavioral (develop healthy lifestyles, learning to organize daily activities better), and environmental factors (increasing parents' awareness and appropriate role modeling for correct internet use).

Theory: Social Cognitive theory.

Duration: Eight 40-80 min sessions/ 3 months

Techniques: self-recognition, self-expression, education, watching videos, playing games, making posters, scheduling Outcome measures: Internet addiction (IAS)

Aim: to assess a curriculum to improve media literacy, so media is used and processed more healthily and therefore, decrease adolescent computer gaming and internet use behavior

Theory: driven by the concept of Media literacy

Duration: Four 90min sessions

Techniques: self-reflection of patterns of use, critical thinking of online content and activities, booklet and online materials use.

Outcome measures: Gaming behavior (KFN-CSAS-II), Internet use (days per week), Addictive internet use (ISS)

Aim: to improve self-control, self-efficacy, internet addiction, and time spent on the internet among middle school students Theory: Social cognitive theory

Duration: Ten 45-minute sessions

Techniques: small group interaction, sharing and feedback, role modeling, case observing, video watching, group practice Outcome measures: Self-control, Self-efficacy, Internet addiction (K-Scale), Time spent on the internet (minutes per day)

Aim: assess the impact of a health education intervention on health behaviors (physical activity, screen time, sugar beverage intake, having breakfast, and Internet Addiction tendency), self-efficacy, and wellbeing

Theory: Social cognitive theory

Duration: Seven two-hour sessions

Techniques: activities that combined knowledge, attitude and practice of behavior.

Outcome measures: Health behaviors, Self -efficacy, Physical activity, Screen time, Internet addiction tendency (YDQ)
All the HBM constructs showed significant changes for the IG after the intervention in the desired direction. The mean score of internet addiction and the prevalence of internet addiction decreased significantly in the intervention group compared to the control group.

CGa: $M=37, S D=2.3, n=80$

IGa: $M=21.9, S D=3.7, n=80$

The intervention group significantly decreased their scores on the IAS after the intervention. This decrease was sustained at the follow-up 9 months later.

CGa: $M=84.91, S D=18.72, n=41$

IGa: $M=76.41, S D=13.85, n=43$

Significant group-time interactions were found for the ISS, showing an increase in the mean for the control group at the three time points measured (baseline, post-test, and follow-up after a year).

CGa: $M=0.41, S D=0.46, n=1039$

IGa: $M=0.34, S D=0.43, n=804$

Self-efficacy and self-control increased significantly in the EG after the intervention and Internet Addiction and time spent on the internet significantly decreased in the intervention group.

CGa: $M=85.37, S D=19.16, n=41$ IGa: $M=61.13, S D=13.23, n=38$

There was a significant reduction in the proportion of students with high screen time and screen addiction tendency in the intervention group. CGa: no $=221(82.2 \%)$, yes $=48$ (17.8\%)

IGa: no = $237(90.1 \%)$, yes $=26(9.9 \%)$

Notes. IAT = Young's Internet Addiction Test, IAS = Internet Addiction Scale, KFN-CSAS-II = German Computer Gaming Addiction Scale, ISS = German Internet Addiction Scale, K-Scale = Internet Addiction Proneness scale, YDQ = Young's diagnostic questionnaire. 
One study was conducted in Germany (Walther et al., 2014), the four other ones in Asia, particularly in China, Iran, South Korea, and Turkey (Maheri et al., 2017; Uysal \& Balci, 2018; S.-Y. Yang \& Kim, 2018; X.-H. Yang et al., 2019). All five of the studies were published after 2014. Three studies targeted adolescents (10-16 years old), the two other ones, college students (16-24 years old). The Iranian study was conducted solely among women, while the rest were conducted in both boys and girls with equivalent proportions among groups. Four studies used a controlled pre-post design, with one group receiving the intervention while the other did not, and outcomes were measured before and after the intervention. In all studies, the participants were randomized to the conditions, but as the randomization was not concealed, some studies took measures to prevent contamination and interviewer bias. All control groups shared similar characteristics to the intervention groups, and were expected to experience similar changes in their environment apart from the intervention. In all cases, groups were tested at baseline to ensure comparability, which resulted in homogeneity tests that revealed no significant differences in the participants' characteristics between groups. The study by Walther et al. (2014) was the only one that used a cluster-level randomized controlled trial (RCT) design, and alerted of slight differences between intervention and control groups in terms of age, socio-economic level, school type, and internet access at baseline.

Three of the programs focused only on reducing IA symptoms (Maheri et al., 2017; Uysal \& Balci, 2018; S.-Y. Yang \& Kim, 2018), one targeted both computer gaming and IA behaviors (Walther et al., 2014), and one targeted internet addiction in the context of a general health behavior intervention also addressing healthy eating, physical activity, stress reduction and healthy sleep (X.-H. Yang et al., 2019). Three of the studies did not provide a specific definition of PIU or IA. However, X.-H. Yang et al. (2019) use the term IA and Young's Diagnostic Questionnaire; S.Y. Yang \& Kim (2018) state that IA is characterized by psychological dependence, tolerance, withdrawal symptoms and provide a reference to DSM-V; and Walther et al. (2014) focus on the relation between digital media use and adolescent mental health in the context of media literacy. Of the two studies that use an explicit definition, Maheri et al. (2017) define IA as an excessive or irrational internet use and make a reference to the criteria of using the internet for more than 38 hours as a sign of addiction proposed by Young (1998). Uysal and Balci (2018, p. 43) define IA as "being incapable of overcoming the desire to go online and the feeling of meaninglessness of the time spent offline, overanxiety and aggression, accompanied by a deterioration in relationships with family, friends, and school life due to excessive use." All of the studies emphasize the pressing need for prevention and report the academic, family, financial, and social consequences of IA.

Four of the programs discussed in the studies were theory-driven, and used techniques and resources from either Social Cognitive Theory or the Health Belief Model. Social cognitive theory states that environmental, personal, and behavioral factors interact in a bi-directional way, in the sense that environmental factors affect the individuals' self-beliefs and drive behavioral change. Among the personal factors, the main concepts that determine behavioral change are self-efficacy and self-regulation. Both the studies by Uysal and Balci (2018) and by S.-Y. Yang and Kim (2018) rely on this theory and include techniques and activities to address environmental factors and personal and behavioral ones. For instance, Uysal and Balci (2018) scheduled interviews with parents and phone calls to track the students' use of the Internet. S.-Y. Yang and Kim (2018) involved the school nurse to follow up with students after the interventions and informed school authorities about IA's serious and harmful effects. Maheri et al. (2017) used the Health Belief Model, which states that health-related behaviors are based on perceived susceptibility (perception of the risk of developing a problem), perceived severity (perception of the seriousness of the consequences), perceived benefits (the belief in the efficacy of the advised action to reduce the risk), perceived barriers (the costs of taking the advised action), cues to action, and self-efficacy. The other programs relied on an educational approach, with one especially involving media literacy (Walther et al., 2014).

In terms of their aims, all of the programs were looking to reduce IA symptoms after the intervention, but they differed in the risk and protective factors addressed. Theory-driven programs relied on change in the levels of the constructs deriving from the theories (e.g., increasing self-efficacy, decreasing perceived barriers), while education-based programs relied on increasing knowledge on internet addiction and its consequences to produce an effect on IA. All programs shared the use of presentations and discussions as techniques to produce the desired change. The most often-used techniques were group discussions and reflection, lectures, video watching, role modeling, and case observing. The length of the interventions varied from 3 to 10 sessions, and all of them were delivered weekly. 
To measure the outcome variables, all programs administered valid and reliable questionnaires measuring $I A$, including Young's Internet Addiction Test (IAT) or more locally developed scales, such as the German Internet Addiction Scale (ISS) or the Internet Addiction Proneness scale (K-Scale). Some studies also measured screen time and internet use using self-reported measures of minutes or hours a day or days per week of internet use.

As for the results, all the interventions reported significant effects in the desired direction. For the theory-driven interventions, significant changes were found for the model-based variables, as well as a significant decrease in IA symptoms in the intervention group compared to the control group after completing the program (Maheri et al., 2017; Uysal \& Balci, 2018; S.-Y. Yang \& Kim, 2018). For the media literacy program, an increase of IA over time was found for the control group, but not for the intervention group, when comparing baseline, post-test, and followup data after one year (Walther et al., 2014). For the health behaviors intervention, a significant reduction was found in the proportion of students with high screen time and IA symptoms after the intervention (X.-H. Yang et al., 2019). It is important to note that three studies (Maheri et al., 2017; Uysal \& Balci, 2018; Walther et al., 2014) collected the post-intervention data three to four months after the intervention took place, while two studies (S.Y. Yang \& Kim, 2018; X.-H. Yang et al., 2019) collected the post-intervention data immediately after the intervention finished. None of the studies sought actively for differences by gender, either in IA symptomatology or in the impact of the intervention.

\section{Meta-Analysis}

Figure 2. Results of Different Meta-Analysis Using a Random Effects Model.

(a)

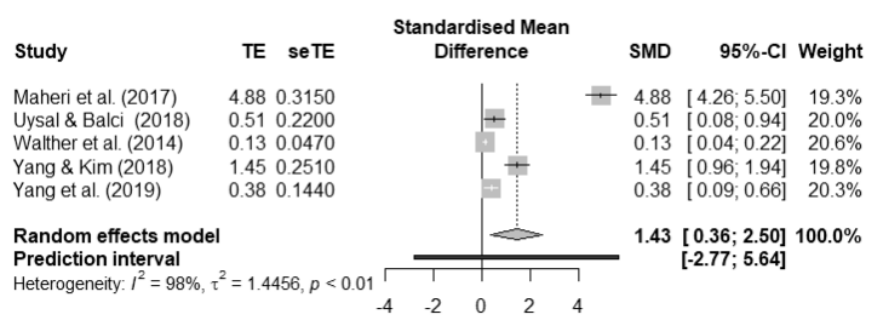

(c)

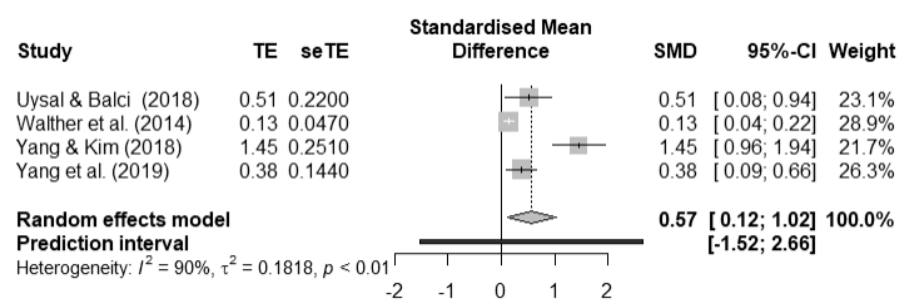

(b)

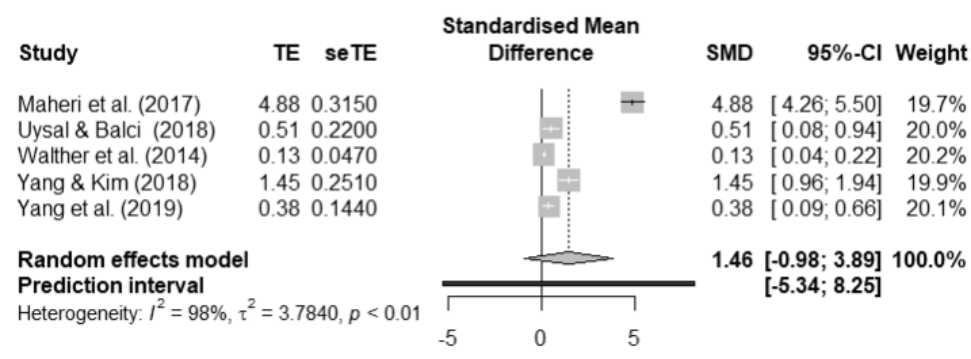

(d)

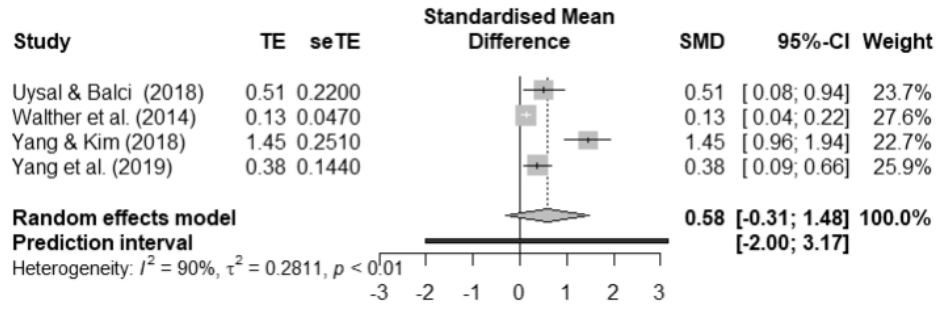

Pooling the five studies samples resulted in a total sample of 965 students in the intervention group and 1201 in the control group. Figure 2 shows the forest plots and statistics for the random-effects model for the pooled posttest effect differences between the groups. Figure 2a displays the results when using the DerSimonian-Laird estimator, which shows a significant overall effect of the interventions. Two studies gave moderate effect sizes, and three high effect sizes. These findings can be considered robust since the confidence intervals did not include zero. The pooled effect sizes favor intervention over no intervention ( $g=1.43,95 \% \mathrm{Cl}[0.36,2.50])$. However, when the HKS estimator is used, more conservative results are obtained, showing no significant combined effect $(95 \%$ $\mathrm{Cl}[0.98,3.89]$, Figure $2 \mathrm{~b})$. Since high levels of heterogeneity were found $\left({ }^{2}=98 \%\right)$, subgroup analysis would be indicated to identify sources of variation, but that is not possible due to the small number of studies. Instead, a leave-one-out analysis was performed to explore the heterogeneity further. As shown in Figure 3, the lowest heterogeneity levels and the lowest overall effects are reached when removing one of the studies (Maheri et al. 2017), which has a disproportionate Hedges' $g$ value. Two re-analyses (Figures $2 c$ and $2 d$ ) involving the four 
remaining studies and summarizing the results of 885 cases in the intervention group and 1121 in the control group gave an overall significant effect when using the DerSimonian-Laird estimator $(g=0.57,95 \% \mathrm{Cl}[0.12,1.02])$ with a prediction interval at a $95 \%$ confidence of -1.52 to 2.66 , but a non-significant effect $(g=0.58,95 \% \mathrm{Cl}[-0.31$, 1.48]) with a prediction interval at a $95 \%$ confidence of -2.00 to 3.17 using the HKSJ estimator. In both cases, heterogeneity remains high at $90 \%$.

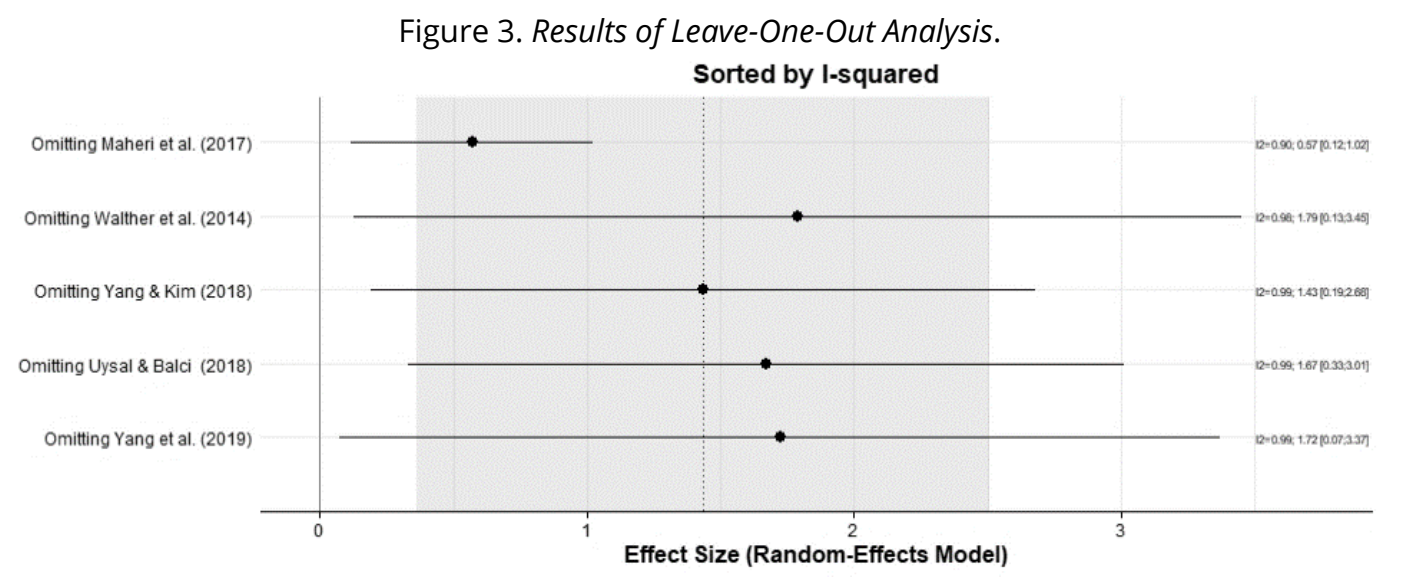

\section{Discussion}

Despite the growing recognition of PIU as an important topic in public health and the acknowledged need for preventive interventions, little is known about the best strategies to prevent this problem or the effectiveness of existing prevention programs. This review aimed to examine the effectiveness of primary prevention programs for PIU in adolescents and youths while also paying attention to the definitions used, the risk and protective factors addressed, and the characteristics of the interventions that are reported in the literature. While precise conceptual and operational definitions of PIU are still lacking, the review integrated studies that aimed to deal with different internet-related problems by considering prevention programs targeting problematic use in general and specific online activities. Such integration is warranted given the commonalities among these behaviors and the fact that restricting the review to one specific online activity would not have given a sufficient number of studies to conduct a meta-analysis.

Although the review did not use the publication date as an inclusion criterion, all of the studies found on this subject were recent, and published since 2014. Moreover, only a small number of studies could be found that focused on primary prevention, although some additional studies were identified that are still ongoing (e.g., Lindenberg et al., 2017; Soto et al., 2018). With one exception, which targeted both online gaming and IA symptoms (Walther et al., 2014), all of the studies targeted PIU and IA in general. While it was expected to find more specific studies by including more search terms aiming for specific behaviors, this was not the case. Based on the excluded studies it can be assumed that studies regarding treatment and secondary or tertiary prevention are more common and larger, which suggests that more efforts are placed on treatment of prevention IA, Internet use disorder, PIU, online gaming, smartphone addiction, and compulsive buying disorder than on prevention (Benson et al., 2014; Chow et al., 2018; Ke \& Wong, 2018a; Lindenberg et al., 2017). In this regard, primary prevention of PIU is an emergent research topic, contrary to treatment.

The studies included in this review mainly portray PIU from an addiction perspective. This is reflected in the definitions given (in those cases when a definition is provided) and the instruments used to assess the outcome variables. While it is understandable that the authors use the best available definitions and tools at hand for conducting and evaluating prevention programs, this perspective disregards the controversial nature of conceptualizing PIU as an addiction and may over-pathologize the condition (Billieux et al., 2015). Although some clinical aspects of PIU may resemble those of addictions, not all PIU behaviors meet the physiological criteria of addiction, such as increasing tolerance, and other PIU features resemble more those of other pathologies. So, it appears that the lack of consensus on how PIU should be conceptualized also transpires in prevention programs. As a result, it is not surprising to see a large variability in the reported outcomes and a high level of heterogeneity in the meta-analysis. 
Concerning the theoretical underpinnings of the prevention programs, three of the five studies included in this review drew on the Social Cognitive Theory as a framework for designing the program and guiding the intervention. As the SCT addresses environmental and personal (i.e., behavioral) factors, the programs based on this theory focus not only on the adolescent but also on parents and the school setting. This "ecological" perspective has become very common in the recent prevention literature (Kwon, 2011; Romano, 2014). For instance, the healthy settings approach to health promotion understands health as created and lived by people within the settings of their everyday life, and therefore, action should be integrated across risk factors in the social context where people engage in daily activities (Dooris et al., 2007; Suárez-Reyes \& Van den Broucke, 2016; World Health Organization, 1986). It is not surprising then that IA prevention programs are conducted in school and university settings. In addition, most of the programs to prevent PIU do not rely solely on education and knowledge exchange to produce the desired change but use a variety of techniques and strategies such as active discussion, reflection, and role modeling. The limited effect of focusing only on knowledge to change behavior has been discussed extensively in the health promotion literature (Pellmar et al., 2002; Ryan, 2009; Van den Broucke, 2014) and has also been acknowledged in preventing internet addiction (Kwon, 2011). Therefore, we believe that future preventive efforts should continue to include the healthy settings perspective and address contextual as well as internal factors, using a variety of techniques addressing multiple determinants of the behavior, rather than providing information only.

While the studies included in this review are of high quality and reported medium to large effect sizes for shortterm effectiveness on their own, they are too few in number to warrant robust conclusions regarding their effectiveness when a pooled effect is considered. For conducting a meta-analysis, a certain amount of heterogeneity is acceptable, and it made sense from a conceptual point of view to combine studies that share enough characteristics and for which sufficient data are available. However, the limited number of studies prevented further exploration of potential sources of variance that may have contributed to the data's high heterogeneity. With more studies, a moderator analysis could be considered to account for the effects of potential moderators such as study design, type of program depending on the underlying theory, culture (especially if including Asian studies), age, and gender. To account for these factors, future reviews could consider increasing the number of studies by including those published in more languages by reducing language restrictions.

Additionally, the low number of studies made it impossible to perform any recommended tests to assess publication bias. The studies that were included favored intervention over no intervention on their own, with medium to high effect sizes, but some had relatively small samples. As studies with significant results and larger effect sizes are more likely to get published than non-significative studies, publication bias cannot be excluded. Additionally, the inclusion of gray literature would have increased the chance of finding studies with nonsignificant effects. Future research should make an effort to include grey literature but continue to include only high-quality studies in the review.

In sum, five studies were found that met our criteria to prevent PIU in general, without specifying specific online behaviors to assess or address, and having the addiction framework as their departing point. Since the conceptualization of IA and PUI continues to be discussed and debated (Baggio et al., 2018; Starcevic \& Aboujaoude, 2017), we believe that prevention efforts should be made for specific problematic behaviors rather than in a general fashion. This will encompass choosing one behavior and accurately conceptualizing it by understanding its phenomenology so that unique risk and protective factors can be detected and addressed. It will also be crucial to develop new measurement tools that consider the behavior's unique nature. Once this is achieved, theory-driven preventive interventions can be developed and, provided rigorous methodologies are used to deliver and evaluate them, meaningful conclusions can be drawn regarding their effectiveness. Only then, reported changes in the behavior could be attributed to the intervention and not to confounding factors or conceptual or methodological limitations.

We believe that the first step towards better research is a rigorous conceptualization of the problematic behavior and its unique features. In the future, efforts to prevent PIU should continue to be carefully designed and theoryinformed, use evidence-based and multiple strategies to address the identified risk and protective factors, and involve relevant stakeholders for the implementation, following the prevention guidelines established for the field of psychology (American Psychological Association, 2014). Moreover, careful attention should be placed on the conceptualization and selection of the behaviors and assessment tools used to measure the outcomes, as they 
are crucial to guide interventions and measure their effectiveness. In the absence of a consensus about these issues, evaluating the effects of intervention remains difficult, and evaluation results will remain inconclusive, while strong evidence is required to inform practitioners and policymakers.

\section{References}

American Psychological Association. (2014). Guidelines for prevention in psychology. The American Psychologist, 69(3), 285-296. https://doi.org/10.1037/a0034569

Anderson, E. L., Steen, E., \& Stavropoulos, V. (2017). Internet use and problematic Internet use: A systematic review of longitudinal research trends in adolescence and emergent adulthood. International Journal of Adolescence and Youth, 22(4), 430-454. https://doi.org/10.1080/02673843.2016.1227716

Bağatarhan, T., \& Siyez, D. M. (2015). Programs for preventing Internet addiction during adolescence: A systematic review. Addicta: The Turkish Journal on Addictions, 4(2), 243-265.

https://doi.org/10.15805/addicta.2017.4.2.0015

Baggio, S., Starcevic, V., Studer, J., Simon, O., Gainsbury, S. M., Gmel, G., \& Billieux, J. (2018). Technology-mediated addictive behaviors constitute a spectrum of related yet distinct conditions: A network perspective. Psychology of Addictive Behaviors, 32(5), 564-572. https://doi.org/10.1037/adb0000379

Benson, A. L., Eisenach, D., Abrams, L., \& van Stolk-Cooke, K. (2014). Stopping overshopping: A preliminary randomized controlled trial of group therapy for compulsive buying disorder. Journal of Groups in Addiction \& Recovery, 9(2), 97-125. https://doi.org/10.1080/1556035X.2014.868725

Billieux, J., Schimmenti, A., Khazaal, Y., Maurage, P., \& Heeren, A. (2015). Are we overpathologizing everyday life? A tenable blueprint for behavioral addiction research. Journal of Behavioral Addictions, 4(3), 119-123.

https://doi.org/10.1556/2006.4.2015.009

Borenstein, M., Hedges, L. V., Higgins, J. P., \& Rothstein, H. R. (2009). Introduction to meta-analysis. John Wiley \& Sons.

Breedvelt, J. J. F., Zamperoni, V., South, E., Uphoff, E. P., Gilbody, S., Bockting, C. L. H., Churchill, R. \& Kousoulis, A. A. (2020). A systematic review of mental health measurement scales for evaluating the effects of mental health prevention interventions. European Journal of Public Health, 30(3), 510-516.

https://doi.org/10.1093/eurpub/ckz233

Chow, K. K. N., Leong, B. D., \& Lee, B. Y. H. (2018). Imagining consequences of excessive smartphone use via a character-based mobile application. International Journal of Mental Health and Addiction, 16(6), 1420-1434. https://doi.org/10.1007/s11469-018-9984-7

DerSimonian, R., \& Laird, N. (1986). Meta-analysis in clinical trials. Controlled Clinical Trials, 7(3), 177-188. https://doi.org/10.1016/0197-2456(86)90046-2

D'Hondt, F., Billieux, J., \& Maurage, P. (2015). Electrophysiological correlates of problematic Internet use: Critical review and perspectives for future research. Neuroscience \& Biobehavioral Reviews, 59, 64-82.

https://doi.org/10.1016/j.neubiorev.2015.10.005

Donner, A., \& Klar, N. (2002). Issues in the meta-analysis of cluster randomized trials. Statistics in Medicine, 21(19), 2971-2980. https://doi.org/10.1002/sim.1301

Dooris, M., Poland, B., Kolbe, L., De Leeuw, E., McCall, D. S., \& Wharf-Higgins, J. (2007). Healthy settings: Building evidence for the effectiveness of whole system health promotion - challenges and future directions. In D. V. McQueen \& C. M. Jones (Eds.), Global perspectives on health promotion effectiveness (pp. 327-352). Springer. 
Elhai, J. D., Dvorak, R. D., Levine, J. C., \& Hall, B. J. (2017). Problematic smartphone use: A conceptual overview and systematic review of relations with anxiety and depression psychopathology. Journal of Affective Disorders, 207, 251-259. https://doi.org/10.1016/j.jad.2016.08.030

EUROSTAT. (2020). Individuals, frequency of Internet use.

https://ec.europa.eu/eurostat/databrowser/view/isoc_ci_ifp_fu/default/table?lang=en

Fineberg, N., Demetrovics, Z., Stein, D. J., Ioannidis, K., Potenza, M. N., Grünblatt, E., Brand, M., Billieux, J., Carmi, L., King, D. L., Grant, J. E., Yücel, M., Dell'Osso, B., Rumpf, H. J., Hall, N., Hollander, E., Goudriaan, A., Menchon, J., Zohar, J., ... Chamberlain, S. (2018). Manifesto for a European research network into problematic usage of the Internet. European Neuropsychopharmacology, 28(11), 1232-1246.

https://doi.org/10.1016/j.euroneuro.2018.08.004

Fontalba-Navas, A., Marin-Olalla, M., Gil-Aguilar, V., Rodriguez-Hurtado, J., Ríos-García, G., \& Pena-Andreu, J. M. (2015). Mental health promotion: Prevention of problematic Internet use among adolescents. Journal of Psychiatry, 18(1), Article 218. https://doi.org/10.4172/Psychiatry.1000218

Gaffney, H., Farrington, D. P., Espelage, D. L., \& Ttofi, M. M. (2019). Are cyberbullying intervention and prevention programs effective? A systematic and meta-analytical review. Aggression and Violent Behavior, 45, 134-153.

https://doi.org/10.1016/j.avb.2018.07.002

Gaysina, G. I., \& Zakirova, V. G. (2017). Prevention of teenager's Internet addiction: Pilot program. Elementary Education Online, 16(4), 1873-1881. http://ilkogretim-online.org.tr/index.php/io/article/view/2199

Griffiths, M. D. (2018). Conceptual issues concerning Internet addiction and Internet gaming disorder: Further critique on Ryding and Kaye (2017). International Journal of Mental Health and Addiction, 16(1), 233-239.

https://doi.org/10.1007/s11469-017-9818-z

Hartung, J., \& Knapp, G. (2001). A refined method for the meta-analysis of controlled clinical trials with binary outcome. Statistics in Medicine, 20(24), 3875-3889. https://doi.org/10.1002/sim.1009

Hedges, L. V., \& Olkin, I. (1984). Nonparametric estimators of effect size in meta-analysis. Psychological Bulletin, 96(3), 573-580. https://doi.org/10.1037/0033-2909.96.3.573

Higgins, J. P. T., \& Green, S. (Eds.). (2008). Cochrane handbook for systematic reviews of interventions. Wiley. https://doi.org/10.1002/9780470712184

Higgins, J. P. T., \& Thompson, S. G. (2002). Quantifying heterogeneity in a meta-analysis. Statistics in Medicine, 21(11), 1539-1558. https://doi.org/10.1002/sim.1186

IntHout, J., loannidis, J. P., \& Borm, G. F. (2014). The Hartung-Knapp-Sidik-Jonkman method for random effects meta-analysis is straightforward and considerably outperforms the standard DerSimonian-Laird method. BMC Medical Research Methodology, 14(1), Article 25. https://doi.org/10.1186/1471-2288-14-25

Joo, A., \& Park, I. (2010). Effects of an empowerment education program in the prevention of internet games addiction in middle school students. Journal of Korean Academy of Nursing, 40(2), 255-263.

https://doi.org/10.4040/jkan.2010.40.2.255

Kardefelt-Winther, D. (2014). A conceptual and methodological critique of internet addiction research: Towards a model of compensatory internet use. Computers in Human Behavior, 31, 351-354.

https://doi.org/10.1016/j.chb.2013.10.059

Kardefelt-Winther, D. (2017a). Conceptualizing Internet use disorders: Addiction or coping process? Psychiatry and Clinical Neurosciences, 71(7), 459-466. https://doi.org/10.1111/pcn.12413 
Kardefelt-Winther, D. (2017b). How does the time children spend using digital technology impact their mental wellbeing, social relationships and physical activity? An evidence-focused literature review (Innocenti Discussion Paper 2017-02). UNICEF Office of Research. https://www.unicef-irc.org/publications/pdf/Children-digital-technologywellbeing.pdf

Kardefelt-Winther, D., Livingstone, S., \& Saeed, M. (2019). Growing up in a connected world (Innocenti Research Report). UNICEF Office of Research. https://www.unicef-irc.org/publications/1060-growing-up-in-a-connectedworld.html

Ke, G. N., \& Wong, S. F. (2018a). A healthy mind for problematic Internet use. Cyberpsychology, Behavior, and Social Networking, 21(10), 637-645. https://doi.org/10.1089/cyber.2018.0072

Ke, G. N., \& Wong, S. F. (2018b). Outcome of the psychological intervention program: Internet use for youth. Journal of Rational-Emotive \& Cognitive-Behavior Therapy, 36(2), 187-200. https://doi.org/10.1007/s10942-0170281-3

Kim, J., LaRose, R., \& Peng, W. (2009). Loneliness as the cause and the effect of problematic Internet use: The relationship between Internet use and psychological well-being. CyberPsychology \& Behavior, 12(4), 451-455. https://doi.org/10.1089/cpb.2008.0327

Kwon J. H. (2011). Toward the prevention of adolescent Internet addiction. In K. S. Young \& C. N. de Abreu (Eds.), Internet addiction: A handbook and guide to evaluation and treatment (pp. 223-243). Wiley.

Lee, H., Seo, M. J., \& Choi, T. Y. (2016). The effect of home-based daily journal writing in Korean adolescents with smartphone addiction. Journal of Korean Medical Science, 31(5), 764-769.

https://doi.org/10.3346/jkms.2016.31.5.764

Leschied, A. W., Saklofske, D. H., \& Flett, G. L. (2018). Handbook of school-based mental health promotion. Springer International Publishing.

Lindenberg, K., Halasy, K., \& Schoenmaekers, S. (2017). A randomized efficacy trial of a cognitive-behavioral group intervention to prevent Internet Use Disorder onset in adolescents: The PROTECT study protocol. Contemporary Clinical Trials Communications, 6, 64-71. https://doi.org/10.1016/j.conctc.2017.02.011

Maheri, A., Tol, A., \& Sadeghi, R. (2017). Assessing the effect of an educational intervention program based on Health Belief Model on preventive behaviors of Internet addiction. Journal of Education and Health Promotion, 6. https://doi.org/10.4103/jehp.jehp_129_15

Marco, C., \& Chóliz, M. (2017). Eficacia de las técnicas de control de la impulsividad en la prevención de la adicción a videojuegos [Effectiveness of impulsivity control techniques to videogame addiction prevention]. Terapia Psicológica, 35(1), 57-69. https://doi.org/10.4067/S0718-48082017000100006

Mihajlov, M., \& Vejmelka, L. (2017). Internet addiction: A review of the first twenty years. Psychiatria Danubina, 29(3), 260-272. https://doi.org/10.24869/psyd.2017.260

Odacı, H., \& Çelik, Ç. B. (2017). Group counselling on college students' Internet dependency and life satisfaction. Journal of Psychologists and Counsellors in Schools, 27(2), 239-250. https://doi.org/10.1017/jgc.2017.9

Pellmar, T. C., Brandt, E. N., Jr., \& Baird, M. A. (2002). Health and behavior: The interplay of biological, behavioral, and social influences: Summary of an Institute of Medicine report. American Journal of Health Promotion, 16(4), 206-219. https://doi.org/10.4278/0890-1171-16.4.206

Romano, J. L. (2014). Prevention in the twenty-first century: Promoting health and wellbeing in education and psychology. Asia Pacific Education Review, 15(3), 417-426. https://doi.org/10.1007/s12564-014-9327-8 
Rumpf, H.-J., Vermulst, A. A., Bischof, A., Kastirke, N., Gürtler, D., Bischof, G., Meerkerk, G.-J., John, U., \& Meyer, C. (2014). Occurence of Internet addiction in a general population sample: A latent class analysis. European Addiction Research, 20(4), 159-166. https://doi.org/10.1159/000354321

Ryan P. (2009). Integrated theory of health behavior change: Background and intervention development. Clinical Nurse Specialist, 23(3), 161-170. https://doi.org/10.1097/NUR.0b013e3181a42373

Shek, D. T. L., \& Leung, H. (2013). Development of an integrated intervention model for Internet addiction in Hong Kong. In A. Tsitsika, M. Janikian, D. E. Greydanus, H. A. Omar, \& J. Merrick (Eds.)., Internet addiction: A public health concern in adolescence (pp. 159-180). Nova Science Publisher's.

Soto, A., de Miguel, N. \& Pérez Díaz, V. (2018). An approach to addiction to new technologies: A proposal for prevention in the school environment and rehabilitation treatment. Psychologist Papers, 39(2), 120-126. https://doi.org/10.23923/pap.psicol2018.2867

Spada, M. M. (2014). An overview of problematic Internet use. Addictive Behaviors, 39(1), 3-6. https://doi.org/10.1016/j.addbeh.2013.09.007

Starcevic, V. (2013). Is Internet addiction a useful concept? Australian \& New Zealand Journal of Psychiatry, 47(1), 16-19. https://doi.org/10.1177/0004867412461693

Starcevic, V., \& Aboujaoude, E. (2017). Internet addiction: Reappraisal of an increasingly inadequate concept. CNS Spectrums, 22(1), 7-13. https://doi.org/10.1017/S1092852915000863

Su, W., Han, X., Jin, C., Yan, Y., \& Potenza, M. N. (2019). Are males more likely to be addicted to the internet than females? A meta-analysis involving 34 global jurisdictions. Computers in Human Behavior, 99, 86-100.

https://doi.org/10.1016/j.chb.2019.04.021

Suárez-Reyes, M., \& Van den Broucke, S. (2016). Implementing the health promoting university approach in culturally different contexts: A systematic review. Global Health Promotion, 23(Suppl. 1), 46-56.

https://doi.org/10.1177/1757975915623933

Tanrikulu, I. (2018). Cyberbullying prevention and intervention programs in schools: A systematic review. School Psychology International, 39(1), 74-91. https://doi.org/10.1177/0143034317745721

Teo, T. (2013). An initial development and validation of a Digital Natives Assessment Scale (DNAS). Computers \& Education, 67, 51-57. https://doi.org/10.1016/j.compedu.2013.02.012

Throuvala, M. A., Griffiths, M. D., Rennoldson, M., \& Kuss, D. J. (2019). School-based prevention for adolescent Internet addiction: Prevention is the key. A systematic literature review. Current Neuropharmacology, 17(6), 507525. https://doi.org/10.2174/1570159X16666180813153806

Tokunaga, R. S. (2010). Following you home from school: A critical review and synthesis of research on cyberbullying victimization. Computers in Human Behavior, 26(3), 277-287.

https://doi.org/10.1016/j.chb.2009.11.014

Uysal, G., \& Balci, S. (2018). Evaluation of a school-based program for Internet addiction of adolescents in Turkey. Journal of Addictions Nursing, 29(1), 43-49. https://doi.org/10.1097/JAN.0000000000000211

Van Den Broucke S. (2014). Needs, norms and nudges: The place of behaviour change in health promotion. Health Promotion International, 29(4), 597-600. https://doi.org/10.1093/heapro/dau099

Viechtbauer, W., \& Cheung, M. W.-L. (2010). Outlier and influence diagnostics for meta-analysis. Research Synthesis Methods, 1(2), 112-125. https://doi.org/10.1002/jrsm.11 
Volpe, U., Dell'Osso, B., Fiorillo, A., Mucic, D., \& Aboujaoude, E. (2015). Internet-related psychopathology: Clinical phenotypes and perspectives in an evolving field. Journal of Psychopathology, 21(4), 406-414.

Vondráčková, P., \& Gabrhelík, R. (2016). Prevention of Internet addiction: A systematic review. Journal of Behavioral Addictions, 5(4), 568-579. https://doi.org/10.1556/2006.5.2016.085

Wallace, P. (2014). Internet addiction disorder and youth. EMBO Reports, 15(1), 12-16. https://doi.org/10.1002/embr.201338222

Walther, B., Hanewinkel, R., \& Morgenstern, M. (2014). Effects of a brief school-based media literacy intervention on digital media use in adolescents: Cluster randomized controlled trial. Cyberpsychology, Behavior, and Social Networking, 17(9), 616-623. https://doi.org/10.1089/cyber.2014.0173

Winkler, A., Dörsing, B., Rief, W., Shen, Y., \& Glombiewski, J. A. (2013). Treatment of internet addiction: A metaanalysis. Clinical Psychology Review, 33(2), 317-329. https://doi.org/10.1016/j.cpr.2012.12.005

World Health Organization. (2015). Public health implications of excessive use of the Internet, computers, smartphones and similar electronic devices: Meeting report.

http://apps.who.int/iris/bitstream/10665/184264/1/9789241509367_eng.pdf

World Health Organization. (1986). Ottawa Charter for Health Promotion. Adopted at an International Conference on Health Promotion - The Move Towards a New Public Health, Nov 17-21, Ottawa, Canada.

https://www.euro.who.int/__data/assets/pdf_file/0004/129532/Ottawa_Charter.pdf

Wu, X., Chen, X., Han, J., Meng, H., Luo, J., Nydegger, L., \& Wu, H. (2013). Prevalence and factors of addictive Internet use among adolescents in Wuhan, China: Interactions of parental relationship with age and hyperactivity-impulsivity. PLoS One, 8(4), Article e61782. https://doi.org/10.1371/journal.pone.0061782

Yang, S.-Y., \& Kim, H.-S. (2018). Effects of a prevention program for internet addiction among middle school students in South Korea. Public Health Nursing, 35(3), 246-255. https://doi.org/10.1111/phn.12394

Yang, X.-H., Yu, H.-J., Liu, M.-W., Zhang, J., Tang, B.-W., Yuan, S., Gasevic, D., Paul, K., Wang, P.-G., \& He, Q.-Q. (2019). The impact of a health education intervention on health behaviors and mental health among Chinese college students. Journal of American College Health, 68(6), 587-592.

https://doi.org/10.1080/07448481.2019.1583659

Young, K. S. (1998). Internet addiction: The emergence of a new clinical disorder. CyberPsychology \& Behavior, 1(3), 237-244. https://doi.org/10.1089/cpb.1998.1.237 


\section{Correspondence to:}

Silvana M. Romero Saletti

Faculté de Psychologie et des Sciences de l'Education

Institut de Recherche en Sciences Psychologiques -IPSY

Université catholique de Louvain

10 Place Cardinal Mercier, bureau D225

Louvain-la-Neuve, 1348

Belgium

Email: silvana.romero(at)uclouvain.be

Editorial record: First submission received on July 9, 2020. Revisions received on November 25, 2020 and February 25, 2021. Accepted for publication on March 1, 2021.

Editor in charge: Lenka Dedkova

\section{About Authors}

Silvana Melissa Romero Saletti is a part time professor at PUCP in Peru and she is currently pursuing her PhD at UCLouvain in Belgium. She holds a MSc in Behavioral and Health Sciences Research from UNED, UAM and UCM, Spain. She is a licensed clinical psychologist from PUCP, Peru. Her research focuses on health prevention and promotion, and the dynamics of behavioral addictions in adolescents and emerging adults.

Stephan Van den Broucke is a professor of public health psychology at UCLouvain in Belgium. His research focuses on preventive behavior change, health promotion, health literacy, patient education and public health capacity building. He published over 100 articles in a variety of high impact peer-reviewed journals and is associate editor of Health Promotion International, as well as an executive board member of the International Union for Health promotion and Education (IUHPE).

Cecilia Chau is a professor of health psychology at the Psychology Department of the PUCP in Peru and a PhD in Psychology from KULeuven. Her research focuses on health risk behaviors in adolescents and university students, with a focus on stress, coping and addictive behaviors. She has published several articles on these topics. 